\author{
E.A. Bakirova ${ }^{1,2}$, Zh.M. Kadirbayeva ${ }^{1,2}$, A.B. Tleulesova ${ }^{3}$ \\ ${ }^{1}$ Institute of mathematics and mathematical modeling SC MES RK, Almaty, Kazakhstan; \\ ${ }^{2}$ Kazakh State Women's Teacher Training University, Almaty, Kazakhstan; \\ ${ }^{3}$ L.N.Gumilyov Eurasian National University, Astana, Kazakhstan \\ (E-mail: bakirova1974@mail.ru, apelman86pm@mail.ru)
}

\title{
On one algorithm for finding a solution to a two-point boundary value problem for loaded differential equations with impulse effect
}

\begin{abstract}
A linear two-point boundary value problem for a system of loaded differential equations with impulse effect is investigated. The parameterization method is used to solve the problem. The essence of parameterization method is that segment, where the loaded differential equation is considered, is divided into parts by loading points, and the initial problem is reduced to the boundary value problem with a parameter. The solution to boundary value problem with parameter is defined as a limit of systems sequence, consisting of the pairs of parameter and function. Parameters are defined by a system of linear algebraic equations. System of linear algebraic equations is determined by the matrices of boundary conditions, the system of loaded differential equations, and the conditions of impulse effect. An algorithm for finding the solution to linear two-point boundary value problem for the systems of loaded differential equations with impulse effect is offered. The convergence conditions of the algorithm providing the existence and uniqueness of solution to the considered problem are established. Sufficient conditions for unique solvability of the problem in the terms of initial data are received.
\end{abstract}

Keywords: boundary value problem, parameterization method, loaded differential equation, impulse effect, algorithm.

In recent years, the interest in studying the loaded differential equations steadily increases, and these equations find numerous applications in practical problems. The intensive research of loaded differential equations with various boundary conditions is observed. The «loaded equation» term has been used in the works of A.M.Nakhushev [1, 2], where the most general definition of loaded equation is given, and various loaded equations are classified in detail, for example, the loaded differential, integral, integro-differential, functional equations, and numerous applications are described. A numerical method for solving the systems of loaded linear nonautonomous ordinary differential equations with non-separated multipoint and integral conditions is offered in [3]. Basic questions in the theory of boundary value problems for loaded differential equations are the same as in the theory of boundary value problems for ordinary differential equations. However, the existence of loaded operator does not always make it possible to apply directly the known theory of boundary value problems [4-6].

Mathematical modeling the evolution of real processes with short-term perturbations, the duration of which can be neglected, leads to the necessity of investigating the differential equations with impulse effect. Various problems for such equations, as well as the methods for their solving and other problems in the theory of impulse systems are considered by many authors [7, 8]. It is known that impulse existence essentially affects the properties of solutions to ordinary differential equations.

Earlier in the works of D.S.Dzhumabaev [9], a parametrization method has been developed for the investigating and solving the two-point boundary value problems for the systems of ordinary differential equations. Parametrization method has allowed establishing the necessary and sufficient conditions for the unique solvability of the problem in the terms of initial data. Based on this method, two-parametrical families of algorithms for finding solutions to the two-point boundary value problems for the systems of ordinary differential equations have been offered. The conditions of feasibility and convergence of those algorithms simultaneously ensure the existence of a unique solution to the problem.

In work [10], the parameterization method is developed for the two-point boundary value problems for the systems of ordinary differential equations with impulse effect, the effective solvability conditions are established, and the constructive algorithms for finding a solution are constructed. Coefficient signs of unique solvability for a linear two-point boundary value problem for the systems of loaded differential equations are found in works [11-15] on the basis of parametrization method, and the algorithms for finding a solution to this problem are constructed. 
The parameterization method in this paper is developed for the linear two-point boundary value problem for the system of loaded differential equations with impulse effect (1)-(3). According to the scheme of parameterization method, the algorithms constructing the approximate solutions to the considered problem are offered. Sufficient conditions for feasibility and convergence of the offered algorithms, as well as for the existence of a unique solution to the two-point boundary value problem for the system of loaded differential equations with impulse effect (1)-(3) are established. One of the basic conditions for the unique solvability of investigated problem is the invertibility of a special matrix compiled from the data of problem.

Consider the linear two-point boundary value problem for the system of loaded differential equations with impulse effect

$$
\begin{gathered}
\frac{d x}{d t}=A_{0}(t) x+\sum_{j=1}^{m} A_{j}(t) \lim _{t \rightarrow \theta_{j}+0} x(t)+f(t), \quad t \in(0, T) \backslash\left\{\theta_{1}, \theta_{2}, \ldots, \theta_{m}\right\} ; \\
B_{0} x(0)+C_{0} x(T)=d, \quad d \in R^{n}, \quad x \in R^{n} ; \\
B_{i} \lim _{t \rightarrow \theta_{i}-0} x(t)-C_{i} \lim _{t \rightarrow \theta_{i}+0} x(t)=\varphi_{i}, \quad \varphi_{i} \in R^{n}, \quad i=\overline{1, m},
\end{gathered}
$$

where the $(n \times n)$-matrices $A_{j}(t), j=\overline{0, m}$, and $n$-vector-function $f(t)$ are piecewise continuous on $[0, T]$ with possible discontinuities of first kind at the points $t=\theta_{i}, \quad i=\overline{1, m}$. Matrices $B_{j}$ and $C_{j}, j=\overline{0, m}$, are $(n \times n)$ constant matrices, $0=\theta_{0}<\theta_{1}<\ldots<\theta_{m}<\theta_{m+1}=T,\|x\|=\max _{i=1, n}\left|x_{i}\right|,\|A(t)\|=\max _{i=1, n} \sum_{j=1}^{n}\left|a_{i j}(t)\right|$.

Solution to problem (1) -(3) is a piecewise continuously differentiable on $[0, T]$ vector function $x(t)$, which satisfies the system of loaded differential equations (1) on $[0, T]$, except the points $t=\theta_{i}, i=\overline{1, m}$, the boundary condition (2) and the conditions of impulse effect at the fixed instants of time (3).

Denote by $P C\left([0, T], \theta_{i}, R^{n}\right)$ the space of piecewise continuous functions with the norm $\|x\|_{1}=\max _{i=\overline{0, m}} \sup _{t \in\left[\theta_{i}, \theta_{i+1}\right)}\|x(t)\|$.

Definition. Problem (1)-(3) is called uniquely solvable, if for any function $f(t) \in P C\left([0, T], \theta_{i}, R^{n}\right)$ and vectors $d \in R^{n}, \varphi_{i} \in R^{n}, \quad i=\overline{1, m}$, it has a unique solution.

Let us consider an example showing that impulse at loading points influences significantly to the property of two-point boundary value problem investigated.

Consider the periodic boundary value problem for the loaded differential equation without impulse effect:

$$
\frac{d x}{d t}=t+x\left(\frac{1}{2}\right), \quad x(0)=x(1), \quad t \in[0,1] .
$$

Obviously, problem (4) has a unique solution $x(t)=\frac{t^{2}}{2}-\frac{t}{2}-\frac{3}{8}$. Along with (4) we consider the periodic boundary value problem for the loaded differential equation with impulse effect

$$
\begin{gathered}
\frac{d x}{d t}=t+x\left(\frac{1}{2}\right), \quad t \in[0,1] \backslash\left\{\frac{1}{2}\right\}, \quad x(0)=x(1) ; \\
4 x\left(\frac{1}{2}+0\right)-x\left(\frac{1}{2}-0\right)=1 .
\end{gathered}
$$

General solution to the equation on $\left[0, \frac{1}{2}\right)$ has the form: $x(t)=\frac{t^{2}}{2}+\frac{t}{4}+2 C t+C, x\left(\frac{1}{2}-0\right)=\frac{1}{4}+2 C$. From (6) we define $x\left(\frac{1}{2}+0\right)$ :

$$
x\left(\frac{1}{2}+0\right)=\frac{1}{4}\left[1+x\left(\frac{1}{2}-0\right)\right]=\frac{1}{4}\left[1+\frac{1}{4}+2 C\right]=\frac{5}{16}+\frac{C}{2} .
$$

Then the solution on $\left[\frac{1}{2}, 1\right]$ takes the form: $x(t)=\frac{t^{2}}{2}+\frac{3}{8} t+C t$.

Now, to make solution satisfied the periodic boundary condition, we need to choose an arbitrary constant $C$ satisfying the equation $C=C+\frac{7}{8}$. Since there is no such number $C$, problem (5), (6) does not have a solution.

Let us now investigate boundary value problem (1)-(3) by the parametrization method. Divide the interval $[0, T]$ into parts by the loading points: $[0, T)=\bigcup_{r=1}^{m+1}\left[\theta_{r-1}, \theta_{r}\right)$. 
Introduce $C\left([0, T], \theta_{r}, R^{n(m+1)}\right)$ as a space of function systems $x[t]=\left(x_{1}(t), x_{2}(t), \ldots, x_{m+1}(t)\right)$, where functions $x_{r}(t), \quad r=\overline{1, m+1}$, are continuous on $\left[\theta_{r-1}, \theta_{r}\right)$ and have finite left-sided limits $\lim _{t \rightarrow \theta_{r}-0} x_{r}(t)$, $r=\overline{1, m+1}$, with the norm $\|x[\cdot]\|_{2}=\max _{r=\overline{1, m+1}} \sup _{t \in\left[\theta_{r-1}, \theta_{r}\right)}\left\|x_{r}(t)\right\|$.

Let $x_{r}(t)$ be the restriction of function $x(t)$ to the $r$-th interval $\left[\theta_{r-1}, \theta_{r}\right), r=\overline{1, m+1}$, i.e. $x_{r}(t)=x(t)$, for $t \in\left[\theta_{r-1}, \theta_{r}\right), r=\overline{1, m+1}$. Introducing the additional parameters $\lambda_{r}=x_{r}\left(\theta_{r-1}\right), r=\overline{1, m+1}$ and performing the replacement $u_{r}(t)=x_{r}(t)-\lambda_{r}$ on each interval $\left[\theta_{r-1}, \theta_{r}\right), r=\overline{1, m+1}$, we obtain the boundary value problem with parameters

$$
\begin{gathered}
\frac{d u_{r}}{d t}=A_{0}(t)\left[u_{r}(t)+\lambda_{r}\right]+\sum_{j=1}^{m} A_{j}(t) \lambda_{j+1}+f(t), \quad t \in\left[\theta_{r-1}, \theta_{r}\right) ; \\
u_{r}\left(\theta_{r-1}\right)=0, \quad r=\overline{1, m+1} ; \\
B_{0} \lambda_{1}+C_{0} \lambda_{m+1}+C_{0} \lim _{t \rightarrow T-0} u_{m+1}(t)=d ; \\
B_{i} \lim _{t \rightarrow \theta_{i}-0} u_{i}(t)+B_{i} \lambda_{i}-C_{i} \lambda_{i+1}=\varphi_{i}, \quad i=\overline{1, m} .
\end{gathered}
$$

Solution to problem $(7)-(10)$ is a pair $(\lambda, u[t])$ with elements $\lambda=\left(\lambda_{1}, \lambda_{2}, \ldots, \lambda_{m+1}\right) \in R^{n(m+1)}$, $u[t]=\left(u_{1}(t), u_{2}(t), \ldots, u_{m+1}(t)\right) \in C\left([0, T], \theta_{r}, R^{n(m+1)}\right)$, where the functions $u_{r}(t)$ are continuously differentiable on $\left[\theta_{r-1}, \theta_{r}\right), \quad r=\overline{1, m+1}$, and for $\lambda_{r}=\lambda_{r}^{*}$ they satisfy the system of ordinary differential equations (7) and conditions (8)-(10).

Problem (1)-(3) is equivalent to problem (7)-(10). So, if the pair $(\tilde{\lambda}, \tilde{u}[t])$ with elements $\tilde{\lambda}=\left(\tilde{\lambda}_{1}, \tilde{\lambda}_{2}, \ldots, \tilde{\lambda}_{m+1}\right) \in R^{n(m+1)}, \tilde{u}[t]=\left(\tilde{u}_{1}(t), \tilde{u}_{2}(t), \ldots, \tilde{u}_{m+1}(t)\right) \in C\left([0, T], \theta_{r}, R^{n(m+1)}\right)$ is a solution to problem (7)-(10), then the function $\tilde{x}(t)$ defined by the equalities $\tilde{x}(t)=\tilde{\lambda}_{r}+\tilde{u}_{r}(t), t \in\left[\theta_{r-1}, \theta_{r}\right)$, $r=\overline{1, m+1}, \tilde{x}(T)=\tilde{\lambda}_{m+1}+\lim _{t \rightarrow T-0} \tilde{u}_{m+1}(t)$ is a solution to the origin boundary value problem (1)-(3). Conversely, if $x(t)$ is a solution to problem (1)-(3), then the pair $(\lambda, u[t])$ with elements $\lambda=$ $=\left(x\left(\theta_{0}\right), x\left(\theta_{1}\right), \ldots, x\left(\theta_{m+1}\right)\right), u[t]=\left(x(t)-x\left(\theta_{0}\right), x(t)-x\left(\theta_{1}\right), \ldots, x(t)-x\left(\theta_{m+1}\right)\right)$ is a solution to problem (7)-(10).

The appearance of initial conditions $u_{r}\left(\theta_{r-1}\right)=0, r=\overline{1, m+1}$, for fixed values of the parameters $\lambda=\left(\lambda_{1}, \lambda_{2}, \ldots, \lambda_{m+1}\right)$ permits to find the functions $u_{r}(t), r=\overline{1, m+1}$, from the Volterra integral equations of the second kind

$$
u_{r}(t)=\int_{\theta_{r-1}}^{t} A_{0}(\tau)\left[u_{r}(\tau)+\lambda_{r}\right] d \tau+\int_{\theta_{r-1}}^{t}\left[\sum_{j=1}^{m} A_{j}(\tau) \lambda_{j+1}+f(\tau)\right] d \tau, t \in\left[\theta_{r-1}, \theta_{r}\right), r=\overline{1, m+1} .
$$

In equation (11), replacing $u_{r}(\tau), r=\overline{1, m+1}$, by the corresponding right-hand side and then repeating this process $\nu(\nu=1,2, \ldots)$ times, we obtain the representation of functions $u_{r}(t), r=\overline{1, m+1}$, in the following form:

$$
u_{r}(t)=H_{\nu, r}^{0}(t) \lambda_{r}+\sum_{j=1}^{m} H_{\nu, r}^{j}(t) \lambda_{j+1}+F_{\nu, r}(t)+G_{\nu, r}\left(u_{r}, t\right), \quad t \in\left[\theta_{r-1}, \theta_{r}\right), \quad r=\overline{1, m+1},
$$

where

$$
\begin{gathered}
H_{\nu, r}^{j}(t)=\int_{\theta_{r-1}}^{t} A_{j}\left(\tau_{1}\right) d \tau_{1}+\ldots+\int_{\theta_{r-1}}^{t} A_{0}\left(\tau_{1}\right) \ldots \int_{\theta_{r-1}}^{\tau_{\nu-2}} A_{0}\left(\tau_{\nu-1}\right) \int_{\theta_{r-1}}^{\tau_{\nu-1}} A_{j}\left(\tau_{\nu}\right) d \tau_{\nu} \ldots d \tau_{1}, j=\overline{0, m} \\
F_{\nu, r}(t)=\int_{\theta_{r-1}}^{t} f\left(\tau_{1}\right) d \tau_{1}+\int_{\theta_{r-1}}^{t} A_{0}\left(\tau_{1}\right) \int_{\theta_{r-1}}^{\tau_{1}} f\left(\tau_{2}\right) d \tau_{2} d \tau_{1}+\ldots+ \\
+\int_{\theta_{r-1}}^{t} A_{0}\left(\tau_{1}\right) \ldots \int_{\theta_{r-1}}^{\tau_{\nu-2}} A_{0}\left(\tau_{\nu-1}\right) \int_{\theta_{r-1}}^{\tau_{\nu-1}} f\left(\tau_{\nu}\right) d \tau_{\nu} \ldots d \tau_{1}
\end{gathered}
$$




$$
G_{\nu, r}(u, t)=\int_{\theta_{r-1}}^{t} A_{0}\left(\tau_{1}\right) \ldots \int_{\theta_{r-1}}^{\tau_{\nu-2}} A_{0}\left(\tau_{\nu-1}\right) \int_{\theta_{r-1}}^{\tau_{\nu-1}} A_{0}\left(\tau_{\nu}\right) u_{r}\left(\tau_{\nu}\right) d \tau_{\nu} \ldots d \tau_{1}, \quad r=\overline{1, m+1} .
$$

By passing in the right-hand side of (12) to the limit as $t \rightarrow \theta_{r}-0, r=\overline{1, m+1}$, and substituting the corresponding expressions into (9) and (10), we obtain the system of equations for the unknown parameters $\lambda_{r}$, $r=\overline{1, m+1}$

$$
\begin{gathered}
B_{0} \lambda_{1}+C_{0}\left[I+H_{\nu, m+1}^{0}(T)\right] \lambda_{m+1}+C_{0} \sum_{j=1}^{m} H_{\nu, m+1}^{i}(T) \lambda_{j+1}= \\
=d-C_{0} F_{\nu, m+1}(T)-C_{0} G_{\nu, m+1}\left(u_{m+1}, T\right) ; \\
B_{i}\left[I+H_{\nu, i}^{0}\left(\theta_{i}\right)\right] \lambda_{i}+B_{i} \sum_{j=1}^{m} H_{\nu, i}^{j}\left(\theta_{i}\right) \lambda_{j+1}-C_{i} \lambda_{i+1}=\varphi_{i}-B_{i} F_{\nu, i}\left(\theta_{i}\right)-B_{i} G_{\nu, i}\left(u_{i}, \theta_{i}\right), \quad i=\overline{1, m},
\end{gathered}
$$

where $I$ is an identity matrix of dimension $(n \times n)$. Denote the matrix corresponding to the left-hand side of system (13), (14) by $Q_{\nu}(\theta)$ and introduce the vectors

$$
\begin{aligned}
& F_{\nu}(\theta)=\left(d-C_{0} F_{\nu, m+1}(T), \varphi_{1}-B_{1} F_{\nu, 1}\left(\theta_{1}\right), \varphi_{2}-B_{2} F_{\nu, 2}\left(\theta_{2}\right), \ldots, \varphi_{m}-B_{m} F_{\nu, m}\left(\theta_{m}\right)\right) ; \\
& G_{\nu}(u, \theta)=\left(C_{0} G_{\nu, m+1}\left(u_{m+1}, T\right), B_{1} G_{\nu, 1}\left(u_{1}, \theta_{1}\right), B_{2} G_{\nu, 2}\left(u_{2}, \theta_{2}\right), \ldots, B_{m} G_{\nu, m}\left(u_{m}, \theta_{m}\right)\right) .
\end{aligned}
$$

Then we rewrite system (13), (14) in the form

$$
Q_{\nu}(\theta) \lambda=F_{\nu}(\theta)-G_{\nu}(u, \theta), \quad \lambda \in R^{n(m+1)} .
$$

Thus, we have the closed system (11), (15) to find the unknown pair $(\lambda, u[t])$, a solution to problem (7)-(10).

Applying the method of successive approximations, we find solution to boundary value problem (7)-(10) and, correspondingly, to boundary value problem (1)-(3). That's an essence of the parameterization method.

Pair $(\lambda, u[t])$, the solution to problem $(7)-(10)$, is found as a limit of the sequence of pairs $\left(\lambda^{(k)}, u^{(k)}[t]\right)$, $k=0,1,2, \ldots$, defined by the following algorithm:

Step 0. ) We assume that for the chosen $\nu \in N$ matrix $Q_{\nu}(\theta)$ is invertible and find initial approximation with respect to the parameter $\lambda^{(0)}=\left(\lambda_{1}^{(0)}, \lambda_{2}^{(0)}, \ldots, \lambda_{m+1}^{(0)}\right) \in R^{n(m+1)}$ from the systems of equations $Q_{\nu}(\theta) \lambda=F_{\nu}(\theta)$, i.e. $\lambda^{(0)}=\left[Q_{\nu}(\theta)\right]^{-1} F_{\nu}(\theta)$. b) Using the components of vector $\lambda^{(0)} \in R^{n(m+1)}$ and solving the Cauchy problems (7), (8) for $\lambda_{r}=\lambda_{r}^{(0)}, \quad r=\overline{1, m+1}$, on the intervals $t \in\left[\theta_{r-1}, \theta_{r}\right), \quad r=\overline{1, m+1}$, we obtain the functions $u_{r}^{(0)}(t), \quad r=\overline{1, m+1}$.

Step 1.) Substituting the found $u_{r}^{(0)}(t), \quad r=\overline{1, m+1}$, into the right-hand side of system (15), we determine $\lambda^{(1)}=\left(\lambda_{1}^{(1)}, \lambda_{2}^{(1)}, \ldots, \lambda_{m+1}^{(1)}\right) \in R^{n(m+1)}$ from $Q_{\nu}(\theta) \lambda=F_{\nu}(\theta)-G_{\nu}\left(u^{(0)}, \theta\right)$. b) Solving the Cauchy problems (7),(8) on the closed intervals $t \in\left[\theta_{r-1}, \theta_{r}\right), r=\overline{1, m+1}$, for $\lambda_{r}=\lambda_{r}^{(1)}, \quad r=\overline{1, m+1}$, we find the functions $u_{r}^{(1)}(t), \quad r=\overline{1, m+1}$, and so on.

Proceeding in this manner, at step $k$ we obtain a pair $\left(\lambda^{(k)}, u^{(k)}[t]\right), k=0,1,2, \ldots$. Note that in part b), for the fixed values of parameter $\lambda_{r}, \quad r=\overline{1, m+1}$, the solution of the Cauchy problem is found separately on each interval $t \in\left[\theta_{r-1}, \theta_{r}\right), r=\overline{1, m+1}$.

Sufficient conditions for the convergence of algorithm, the existence of a unique solution to the linear twopoint boundary value problem for the system of loaded differential equations with impulse effect (1)-(3), are provided by the following assertion:

Theorem. Suppose that for some $\nu \in N$, the matrix $Q_{\nu}(\theta): R^{n(m+1)} \rightarrow R^{n(m+1)}$ is invertible, and the following inequalities are true:

$$
\begin{gathered}
\left\|\left[Q_{\nu}(\theta)\right]^{-1}\right\| \leq \gamma_{\nu}(\theta) ; \\
q_{\nu}(\theta)=\gamma_{\nu}(\theta) \max \left[1, \max _{i=\overline{1, m}}\left\|B_{i}\right\|,\left\|C_{0}\right\|\right]\left\{e^{\alpha_{0} h}-\sum_{i=0}^{\nu} \frac{\left(\alpha_{0} h\right)^{i}}{i !}+h \sum_{j=1}^{m} \alpha_{j}\left[e^{\alpha_{0} h}-\sum_{i=0}^{\nu-1} \frac{\left(\alpha_{0} h\right)^{i}}{i !}\right]\right\}<1,
\end{gathered}
$$

where $h=\max _{r=\overline{1, m+1}}\left(\theta_{r}-\theta_{r-1}\right),\left\|A_{i}(t)\right\| \leq \alpha_{i}, i=\overline{0, m}$.

Then the linear two-point boundary value problem for the system of loaded differential equations with impulse effect (1)-(3) has a unique solution. 
The proof of Theorem with minor changes is similar to the proof of Theorem 3.2 in [10].

To illustrate the Theorem, we consider the following example. On $[0,1]$ for the system of loaded differential equations

$$
\frac{d x}{d t}=\left(\begin{array}{cc}
0 & \frac{1}{2} \\
\frac{t}{4} & 0
\end{array}\right) x+\left(\begin{array}{cc}
\frac{1}{8} & 0 \\
0 & \frac{t}{16}
\end{array}\right) x\left(\frac{1}{2}\right)+\left(\begin{array}{c}
f_{1}(t) \\
f_{2}(t)
\end{array}\right), \quad t \in[0,1] \backslash\left\{\frac{1}{2}\right\}
$$

we consider the linear two-point boundary value problem with impulse effect

$$
\begin{gathered}
\left(\begin{array}{ll}
1 & 0 \\
0 & 1
\end{array}\right) x(0)+\left(\begin{array}{cc}
-1 & 0 \\
0 & -1
\end{array}\right) x(1)=\left(\begin{array}{l}
d_{1} \\
d_{2}
\end{array}\right) \\
\left(\begin{array}{ll}
1 & 0 \\
0 & 2
\end{array}\right) \lim _{t \rightarrow \frac{1}{2}-0} x(t)-\left(\begin{array}{cc}
2 & 1 \\
-1 & 0
\end{array}\right) \lim _{t \rightarrow \frac{1}{2}+0} x(t)=\left(\begin{array}{l}
\varphi_{1} \\
\varphi_{2}
\end{array}\right) .
\end{gathered}
$$

Divide the segment $[0,1]$ into two parts: $[0,1)=[0,1 / 2) \bigcup[1 / 2,1)$. Introducing the additional parameters $\lambda_{1}=x(0), \lambda_{2}=x_{2}(1 / 2)$, we pass to the boundary value problem with parameters

$$
\begin{gathered}
\frac{d u_{r}}{d t}=\left(\begin{array}{cc}
0 & \frac{1}{2} \\
\frac{t}{4} & 0
\end{array}\right)\left[u_{r}+\lambda_{r}\right]+\left(\begin{array}{cc}
\frac{1}{8} & 0 \\
0 & \frac{t}{16}
\end{array}\right) \lambda_{2}+\left(\begin{array}{c}
f_{1}(t) \\
f_{2}(t)
\end{array}\right), \quad r=\overline{1,2}, \\
u_{1}(0)=0, \quad u_{2}(1 / 2)=0, \\
\left(\begin{array}{ll}
1 & 0 \\
0 & 1
\end{array}\right) \lambda_{1}+\left(\begin{array}{cc}
-1 & 0 \\
0 & -1
\end{array}\right) \lambda_{2}+\left(\begin{array}{cc}
-1 & 0 \\
0 & -1
\end{array}\right) \lim _{t \rightarrow 1-0} u_{2}(t)=\left(\begin{array}{c}
d_{1} \\
d_{2}
\end{array}\right) ; \\
\left(\begin{array}{ll}
1 & 0 \\
0 & 2
\end{array}\right) \lambda_{1}+\left(\begin{array}{cc}
1 & 0 \\
0 & 2
\end{array}\right) \lim _{t \rightarrow \frac{1}{2}-0} u_{1}(t)-\left(\begin{array}{cc}
2 & 1 \\
-1 & 0
\end{array}\right) \lambda_{2}=\left(\begin{array}{c}
\varphi_{1} \\
\varphi_{2}
\end{array}\right) .
\end{gathered}
$$

For $\nu=1$ matrix $Q_{1}(\theta)$ has the next form:

$$
Q_{1}(\theta)=\left(\begin{array}{cccc}
1 & 0 & -1.0625 & -0.25 \\
0 & 1 & -0.09375 & -1.02344 \\
1 & 0.25 & -1.9375 & -1 \\
0.0625 & 2 & 1 & 0.01563
\end{array}\right)
$$

Matrix $Q_{1}(\theta)$ is invertible and

$$
\left[Q_{1}(\theta)\right]^{-1}=\left(\begin{array}{cccc}
l 2.66431 & 0.95466 & -1.64735 & -0.27141 \\
-0.9913 & -0.67168 & 0.94646 & 0.71753 \\
1.83385 & 1.31109 & -1.80699 & -0.42967 \\
-1.13659 & -1.75349 & 1.09031 & 0.74046
\end{array}\right)
$$

Let us verify the implementation of the Theorem conditions:

$$
\begin{gathered}
\left\|\left[Q_{1}(\theta)\right]^{-1}\right\| \leq 5.53773 \\
q_{1}(\theta)=5.53773 \cdot \max \left[1,\left\|B_{1}\right\|,\left\|C_{0}\right\|\right] \cdot\left[e^{0.25}-1-0.25+0.125 \cdot 0.5 \cdot\left(e^{0.25}-1\right)\right]=0.57345<1 .
\end{gathered}
$$

Thus, all assumptions of Theorem are true, and problem (18)-(20) has a unique solution.

\section{References}

1 Нахушев А.М. Нагруженные уравнения и их применение / А.М. Нахушев. - М.: Наука, 2012. 232 c.

2 Нахушев А.М. Уравнения математической биологии / А.М. Нахушев. - М.: Высш. шк., 1995. 205 c. 
3 Абдуллаев В.М. О численном решении нагруженных дифференциальных уравнений / В.М. Абдуллаев, К.Р. Айда-заде // Журнал вычислительной математики и математической физики. - 2004. Т. 44, № 9. - С. 1585-1595.

4 Нахушев А.М. Об одном приближенном методе решения краевых задач для дифференциальных уравнений и его приложения к динамике почвенной влаги грунтовых вод / А.М. Нахушев // Дифференциальные уравнения. - 1982. - Т. 18, № 1. - С. 72-81.

5 Dzhenaliev M.T. Loaded Equations with Periodic Boundary Conditions. Differential Equations. - 2001. - Vol. 37, No. 1. - P. 51-57.

6 Дженалиев М.Т. Нагруженные уравнения как возмущения дифференциальных уравнений М.Т. Дженалиев, М.И. Рамазанов. - Алматы: Ғылым, 2010. - 334 с.

7 Lakshmikantham V. Theory of impulsive differential equations / V. Lakshmikantham, D. Bainov, P. Simonov. - Singapore: World Scientific Publ., 1989. - 273 p.

8 Samoilenko A.M. Impulsive Differential Equations / A.M. Samoilenko, N.A. Perestyuk. - Singapore: World Scientific Publ., 1995. - 462 p.

9 Джумабаев Д.С. Признаки однозначной разрешимости линейной краевой задачи для обыкновенного дифференциального уравнения / Д.С. Джумабаев // Журнал вычислительной математики и математической физики. - 1989. - Т. 29, № 1. - С. 50-66.

10 Тлеулесова А.Б. Об однозначной разрешимости двухточечной краевой задачи с импульсным воздействием / А.Б. Тлеулесова // Математический журнал. - 2004. - Т. 4, № 4. - С. 93-102.

11 Бакирова Э.А. О признаке однозначной разрешимости двухточечной краевой задачи для системы нагруженных дифференциальных уравнений / Э.А.Бакирова // Известия НАН РК. Серия физикоматематическая. - 2005. - № 1. - С. 95-102.

12 Джумабаев Д.С. Об одной численной реализации метода параметризации решения линейной краевой задачи для нагруженного дифференциального уравнений / Д.С. Джумабаев, Г.Б. Илиясова // Известия НАН РК. Серия физико-математическая. - 2014. - № 2. - С. 275-280.

13 Бакирова Э.А. О необходимых и достаточных условиях однозначной разрешимости двухточечной краевой задачи для нагруженных дифференциальных уравнений / Э.А. Бакирова // Математический журнал. - 2005. - Т. 5, № 3. - С. 25-34.

14 Кадирбаева Ж.М. Об одном алгоритме нахождения решения линейной двухточечной краевой задачи для нагруженных дифференциальных уравнений / Ж.М. Кадирбаева // Математический журнал. - 2009. - Т. 9, № 2. -C. 64-70.

15 Akzhigitov E.A. On a solvability of two-point boundary value problem for loaded differential equations. Science review. S.Seifullin Kazakh Agro Technical University / E.A.Akzhigitov, Zh.M.Kadirbayeva. 2012. - No. 2. - P. 35-40.

\author{
Э.А. Бакирова, Ж.М. Кадирбаева, А.Б. Тлеулесова
}

\title{
Импульстік әсері бар жүктелген дифференциалдық теңдеулер үшін екінүктелі шеттік есептің шешімін табудың алгоритмі туралы
}

\begin{abstract}
Импульстік әсері бар жүктелген дифференциалдық теңдеулер жүйесі үшін сызықты екінүктелі шеттік есеп зерттелді. Қарастырылып отырған есепті шешу үшін параметрлеу әдісі қолданылды. Параметрлеу әдісінің маңызы жүктелген дифференциалдық теңдеулер жүйесі қарастырылып отырған кесінді жіктеу нүктелерімен бөліктерге бөлінеді және бастапқы есеп параметрі бар пара пар шеттік есепке келтірілді. Параметрі бар шеттік есептің шешімі параметр және функция жұптар жүйесі тізбегінің шегі ретінде анықталды. Параметрлер шеттік шарт пен импульстік әсер шарты матрицалары және жүктелген дифференциалдық теңдеулер жүйесі арқылы анықталатын сызықты алгебралық теңдеулер жүйесінен табылды. Импульстік әсері бар жүктелген дифференциалдық теңдеулер жүйесі үшін сызықты екінүктелі шеттік есептің шешімін табудың алгоритмі ұсынылды. Зерттеліп отырған
\end{abstract}


есептің шешімінің бар болуы мен жалғыздығын қамтамасыз ететін ұсынылған алгоритмнің жинақтылығының шарттары тағайындалған. Есептің бірмәнді шешілімділігінің жеткілікті шарттары бастапқы берілімдер терминінде алынған.

Kiлm сөздер: өлкелік міндет, параметр әдісі, жүктелген дифференциалдық теңдеулер, импульстік әсері, алгоритм.

\author{
Э.А. Бакирова, Ж.М. Кадирбаева, А.Б. Тлеулесова
}

\title{
Об одном алгоритме нахождения решения двухточечной краевой задачи для нагруженных дифференциальных уравнений с импульсным воздействием
}

\begin{abstract}
Исследована линейная двухточечная краевая задача для системы нагруженных дифференциальных уравнений с импульсным воздействием. Для решения рассматриваемой задачи был применен метод параметризации. Суть метода параметризации заключается в том, что отрезок, где рассматривается нагруженное дифференциальное уравнение, разбивается на части точками нагружения, и исходная задача сводится к эквивалентной краевой задаче с параметром. Решение краевой задачи с параметром определяется как предел последовательности систем пар параметра и функции. Параметры находятся из системы линейных алгебраических уравнений, определяемых по матрицам системы нагруженных дифференциальных уравнений, краевого условия и условия импульсного воздействия. Предложен алгоритм нахождения решения линейной двухточечной краевой задачи для систем нагруженных дифференциальных уравнений с импульсным воздействием. Установлены условия сходимости предложенного алгоритма, обеспечивающие существование и единственность решения исследуемой задачи. Получены достаточные условия однозначной разрешимости задачи в терминах исходных данных.
\end{abstract}

Ключевые слова: краевая задача, метод параметризации, нагруженное дифференциальное уравнение, импульсный эффект, алгоритм.

\section{References}

1 Nakhushev, A.M. (2012). Nahruzhennye uravneniia i ikh primenenie [Loaded equations and applications]. Moscow: Nauka [in Russian].

2 Nakhushev, A.M. (1995). Uravneniia matematicheskoi biolohii [Equations of Mathematical Biology]. Moscow: Vyschaya shkola [in Russian].

3 Abdullaev, V.M. \& Aida-zade, K.R. (2004). O chislennom reshenii nahruzhennykh differentsialnykh uravnenii [On a numerical solution of loaded differential equations]. Zhurnal vychislitelnoi matematiki $i$ matematicheskoi fiziki - Journal of computational mathematics and mathematical physics, Vol. 44, 9, 1585-1595 [in Russian].

4 Nakhushev, A.M. (1982). Ob odnom priblizhennom metode resheniia kraevykh zadach dlia differentsialnykh uravnenii i eho prilozheniia $\mathrm{k}$ dinamike pochvennoi vlahi hruntovykh vod [An approximation method for solving boundary value problems for differential equations with applications to the dynamics of soil moisture and groundwater]. Differentsialnye uravneniia - Differential equations, Vol. 18, 1, 72-81 [in Russian].

5 Dzhenaliev, M.T. (2001). Loaded Equations with Periodic Boundary Conditions. Differential Equations, Vol. 37, 1, 51-57.

6 Dzhenaliev, M.T. \& Ramazanov, M.I. (2010). Nahruzhennye uravneniia kak vozmushcheniia differentsialnykh uravnenii [Loaded equations are as perturbation of differential equations]. Almaty: Gylym [in Russian]

7 Lakshmikantham, V., Bainov, D. \& Simonov, P. (1989). Theory of impulsive differential equations. Singapore: World Scientific Publ.

8 Samoilenko, A.M. \& Perestyuk, N.A. (1995). Impulsive Differential Equations. Singapore: World Scientific. 
9 Dzhumabaev, D.S. (1989). Priznaki odnoznachnoi razreshimosti lineinoi kraevoi zadachi dlia obyknovennoho differentsialnoho uravneniia [Conditions of the unique solvability of a linear boundary value problem for ordinary differential equation]. Zhurnal vychislitelnoi matematiki i matematicheskoi fiziki - Journal of computational mathematics and mathematical physics, Vol. 29, 1, 50-66 [in Russian].

10 Tleulesova, A.B. (2004). Ob odnoznachnoi razreshimosti dvukhtochechnoi kraevoi zadachi s impulsnym vozdeistviem [On an unique solvability of a two-point boundary value problem of impulse effect]. Matematicheskii zhurnal - Mathematical Journal, Vol. 4, 4, 93-102 [in Russian].

11 Bakirova, E.A. (2005). O priznake odnoznachnoi razreshimosti dvukhtochechnoi kraevoi zadachi dlia sistemy nahruzhennykh differentsialnykh uravnenii [ On a criterion of the unique solvability of a two-point boundary value problem for loaded differential equations]. Izvestiia NAN RK. Seria phisiko-matematicheskaia - News NAS RK. Seria physics and mathematics, 1, 95-102 [in Russian].

12 Dzhumabaev, D.S. \& Iliyassova, G.B. (2014). Ob odnoi chislennoi realizatsii metoda parametrizatsii resheniia lineinoi kraevoi zadachi dlia nahruzhennoho differentsialnoho uravneniia [On one numerical implementation of the parameterization method for solving of linear boundary value problem for loaded differential equations]. Izvestiia NAS RK. Seriia phisiko-mathematicheskaia - News NAS RK. Seria physics and mathematics, 2, 275-280 [in Russian].

13 Bakirova, E.A. (2005). O neobkhodimykh i dostatochnykh usloviyakh odnoznachnoi razreshimosti dvukhtochechnoi kraevoi zadachi dlia nahruzhennykh differentsialnykh uravnenii [On necessary and sufficient conditions of the unique solvability of a two-point boundary value problem for loaded differential equations]. Matematicheskii zhurnal - Mathematical Journal, Vol. 5, 3, 25-34 [in Russian].

14 Kadirbayeva, Zh.M. (2009). Ob odnom alhoritme nakhozhdeniia resheniia lineinoi dvukhtochechnoi kraevoi zadachi dlia nahruzhennykh differentsialnykh uravnenii [An algorithm for finding the solution of linear two-point boundary value problem for loaded differential equations]. Matematicheskii zhurnal - Mathezmatizcal Journal, Vol. 9, 2, 64-70 [in Russian].

15 Akzhigitov, E.A. \& Kadirbayeva, Zh.M. (2012). On a solvability of two-point boundary value problem for loaded differential equations. Science review. S.Seifullin Kazakh Agro Technical University, 2, 35-40. 